\title{
Paracoccidioidomycosis in Childhood - A Case Report
}

\author{
Mônica Silva Costa Janson Ney*1, Rebeca Moraes Ielo Cruz ${ }^{1}$, Cristiane Tamily Nishimoto ${ }^{1}$ and César de Souza Bastos \\ Júnior $^{2}$
}

${ }^{1}$ Radiology's Service, Marcilio Dias Navy Hospital (HNMD), Brazil

${ }^{2}$ Pathologic's Service, Marcilio Dias Navy Hospital (HNMD), Brazil

Received: 畊: December 18, 2018; Published: 制: January 04, 2019

*Corresponding author: Mônica Silva Costa Janson Ney, Rua Cesar Zama 185- 3o andar, Departamento de Radiologia, Brazil

\begin{abstract}
Paracoccidioidomycosis is a systemic mycosis originating in Latin America and endemic in some regions of Brazil. It occurs mainly among rural populations, predominantly affecting males with age about 30 and 60 years old. It is infrequent below 14 years old. The etiologic agent is a thermodymorphic fungus denominated Paracoccidioides brasiliensis. The infection occurs by inhalation the fungus spores, initially involving the upper airways and the lung. Due to the hematogenous or lymphatic dissemination, the lymphatic system, the adrenal glands, the bones, the digestive tract and the central nervous system can also be affected. Imaging methods, especially CT and ultrasound, allow a better evaluation of the alterations found in the disseminated disease, which in most cases are nonspecific, and may simulate both neoplastic lesions and chronic infections, and are often indistinguishable from tuberculosis.

The definitive diagnosis was obtained through a lesion tissue biopsy that demonstrated infection by the fungus. In this case report, paracoccidioidomycosis had an unusual presentation in a 6 years old child with non-specific gastrointestinal symptoms associated with palpable abdominal mass. Ultrasonography and computed tomography showed lymph node enlargement in the abdomen and pelvis. The diagnosis was obtained by pelvic lymph node biopsy that demonstrated the infection by Paracoccidioides. The treatment was made with amphotericin B for 28 days during the hospital internment. After clinical and radiological improvement, she was discharged from hospital with Itraconazole for another 28 days.
\end{abstract}

Keywords: Paracoccidioidomycosis; Childhood; CT; Ultrasonography; Tissue biops

\section{Introduction}

Paracoccidioidomycosis is a systemic mycosis described by Adolfo Lutz in 1908, also called Lutz's disease, South American blastomycosis, Brazilian blastomycosis, Lutz Splendore-Almeida disease and Lutz's mycosis, whose etiologic agent is a thermodymorphic fungus denominated Paracoccidioides brasiliensis. It originates in Latin America, and its highest incidence is recorded in South America, notably in Brazil, Argentina, Colombia and Venezuela. In Brazil, the disease is endemic in the states of São Paulo, Rio de Janeiro, Minas Gerais, Rio Grande do Sul and Mato Grosso [1], mainly among rural populations, affecting males about 30 to 60 years-old. The annual incidence in rural endemic areas in Brazil ranges from 3-4 new cases / 1,000,000 inhabitants to 1-3 new cases / 100,000 inhabitants [2]. It is considered the third cause of death by chronic infectious disease, resulting in a mortality rate of 1.65 cases / $1,000,000$ inhabitants.

The incidence of paracoccidioidomycosis is higher among men about 1:10-15 men. It is infrequent below the age of 14 years old, and there is no predominance of gender in this age group [2] In the reported clinical case the disease affected a child under 7 years-old, which occurs very rarely, but the clinical findings were compatible with the acute / subacute childhood / adolescence form of the disease. Because it is not a common pathology in this age group, paracoccidioidomycosis did not enter as a differential diagnosis. The definitive diagnosis was made by lymph node biopsy that demonstrated fungal structures suspicious of Paracoccidioides. This study was approved by the committee of ethics in research of the Marcilio Dias Navy Hospital and was waived the application of the written informed consent.

\section{Clinical Case}

A 6-year-old female child, living in the Green Coast of Rio de Janeiro state, Brazil, was admitted in the pediatric emergency department with a clinical condition of constipation, fever and asthenia that began 48 hours before. At physical examination, she was stained, hydrated, anicteric and without palpable lymph node 
enlargement. Abdomen was globose, tense, with palpable liver $4 \mathrm{~cm}$ from costal border, and with palpable movable mass on right flank, somewhat hardened and painless. She was a previously healthy child. According to the mother's report, the child had been bitten by a tick 72 hours before the onset of symptoms, with no other events abnormal to the child's routine. Laboratory tests performed at admission revealed: Hemogram: Hb 10g\%; Ht 30.6\%; leukocytes 20,300 cels.; eosinophils 46\%; Platelets 664,000; Urea $13.4 \mathrm{mg} / \mathrm{dl}$; Creatinine $0.4 \mathrm{mg} / \mathrm{dl}$; Total Proteins $9.6 \mathrm{~g} \%$; Albumin 2.3g\%; TGO 26 U / l; TGP 44 U / l; Total bilirubin 0.7 mg / dl; Direct bilirubin 0.4 mg / dl; Indirect bilirubin $0.3 \mathrm{mg} / \mathrm{dl}$; Alkaline phosphatase 735.2 U / l; Glucose 52.8 mg / dl; gammaGT 646.3; Amylase 17.5 mg / dl; Lipase $5.1 \mathrm{mg} / \mathrm{dl}$; negative urine analysis and urinoculture.

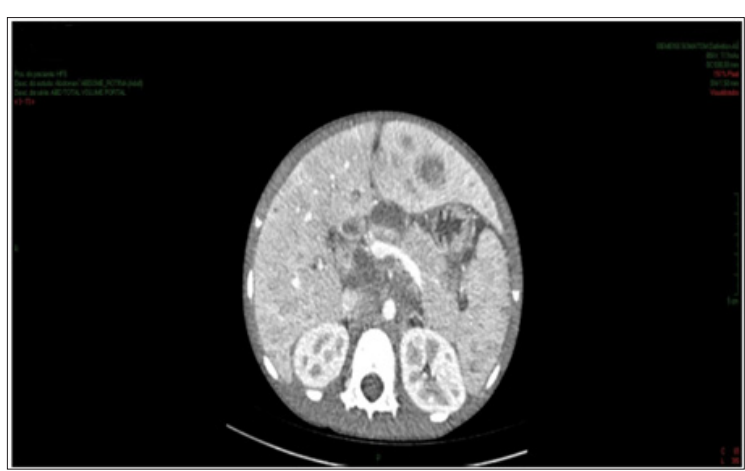

Figure 1: Contrast-enhanced abdomen CT showing increased dimensions of the liver with heterogeneous density at the expense of diffuse hypodense images. Spleen enlarged and heterogeneous.

Enhanced abdominal CT performed on the $1^{\text {st }}$ day of hospitalization, revealed enlarged liver, with heterogeneous attenuation, at the expense of diffuse, ill-defined, sparse hypodense images, measuring up to $2.4 \mathrm{~cm}$ in segment II. Mild dilation of the intrahepatic bile ducts. Spleen enlarged, heterogeneous texture, at the expense of diffuse hypodense images. A hypodense, amorphous, poorly delimited (Figure 1). Expansive formation located in retroperitoneum and mesenteric root with involvement of the mesenteric vessels, without determining stenosis of the same, consistent with lymph node conglomerate. Prominent para-aortic lymph nodes, measuring up to $2.6 \times 1.4 \mathrm{~cm}$ (Figure 2). Moderate amount of free fluid in the abdominal cavity. Hypodense and hypocaptant mass of ill-defined limits on the right flank, compatible with lymph node mass (Figure 3 ). In chest CT no lymph node enlargement was been in the mediastinum. On the 3rd day of hospitalization, abdominal ultrasonography was also performed to corroborate the CT findings, evidencing an enlarged liver, diffusely heterogeneous with small hypoechoic solid nodules distributed in the hepatic parenchyma. Heterogeneous pancreas, with presence of solid hypoechoic nodule, adjacent to the body, measuring 2.3 $\mathrm{cm}$, suggesting lifonodomegaly. Enlarged spleen with presence of multiple hypoechoic solid nodules of small dimensions. Moderate ascites. Solid mass on the right flank measuring approximately 10 $\mathrm{cm}$ suggestive of confluent lymph nodes. The left inguinal lymph node biopsy was analyzed by the simple "imprint" and "cell block", the macroscopy of which describes a brownish, lobed and unctuous fragment measuring $1.3 \times 0.6 \times 0.4 \mathrm{~cm}$. Microscopy showed a chronic/acute granulomatous inflammatory process containing multinucleated giant cells in the lymph node tissue. Special coloring by the PAS and Grocott method evidenced fungal structures suspected of Paracoccidioides (Figures 4 \& 5).

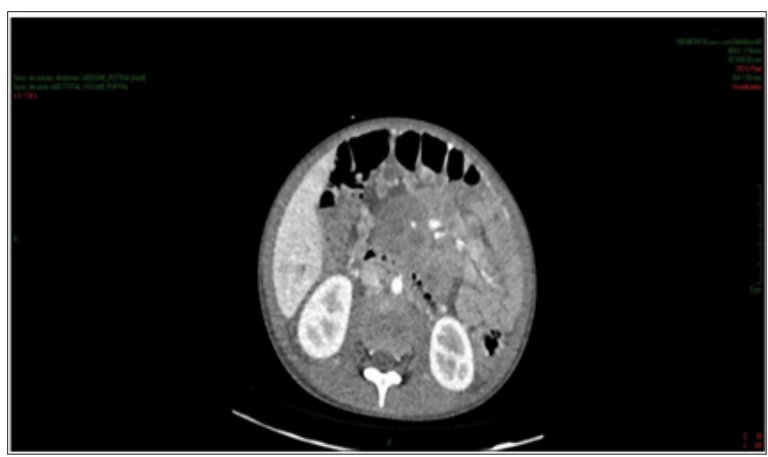

Figure 2: Contrast-enhanced abdomen CT showing expansive formation in the retroperitoneum and root of the mesentery compatible with lymph node conglomerate. Numerous and prominent para-aortic lymph nodes.

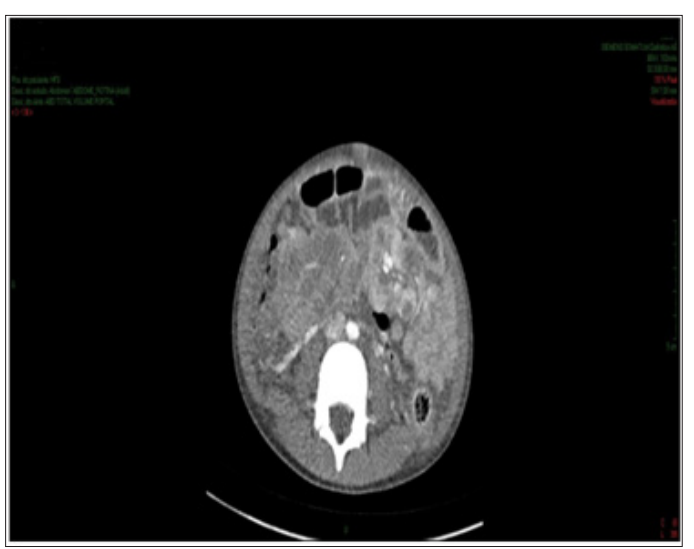

Figure 3: Contrast-enhanced abdomen CT showing hypodense, hypocaptant mass of ill-defined limits on the right flank, compatible with lymph node mass.

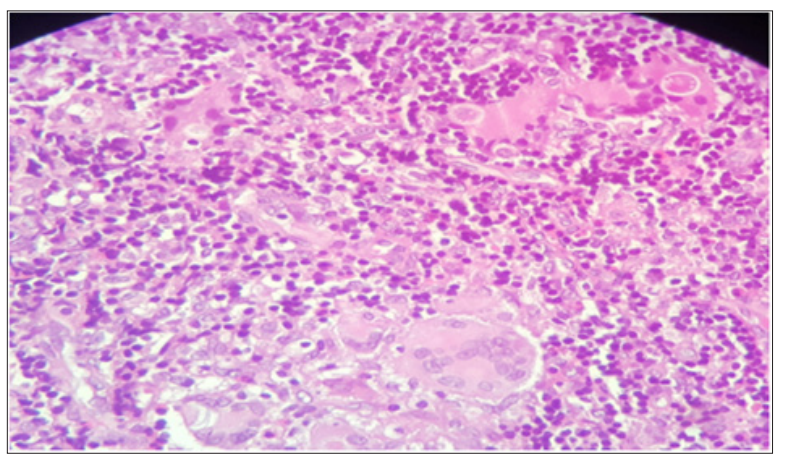

Figure 4: HE 400X Multinucleated giant cells phagocytizing fungal structures. 


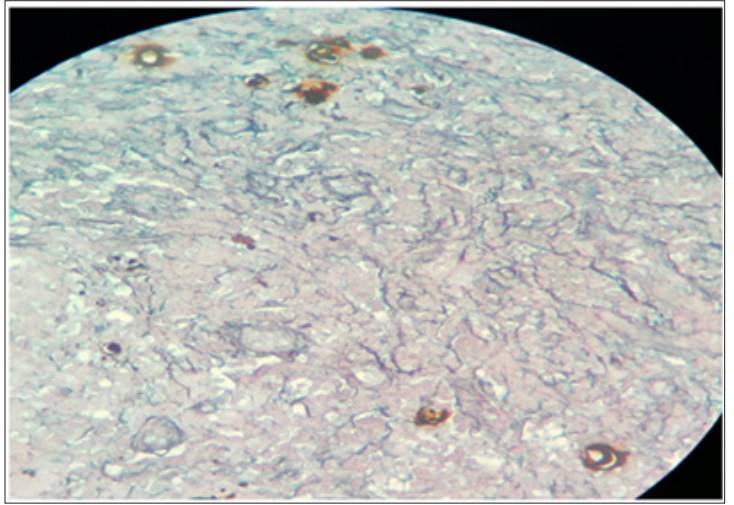

Figure 5: Silver staining 400X showing the fungal structures.

Patient started treatment with amphotericin B and after 15 days performed a new ultrasound of the abdomen to control treatment that showed persistence of lymph node enlargement near to the pancreas body, reduction of ascites, with a small amount of free fluid in the pelvic cavity, and a volumetric reduction of the linfonodal mass, located on the right flank measuring $4.7 \times 4.0 \mathrm{~cm}$ (Figure 6). During the 41 days of hospitalization, the patient remained hemodynamically stable, presenting febrile peaks attributed to the intercurrence of infectious condition caused by K.pneumoniae and S.hominis being treated with cefepime and vancomycin. There were bilaterally microadenomegalies in the posterior cervical chain and right axillary adenomegaly, mobile, painless and elastic, with no other conditions. Patient evolved with clinical, laboratory and radiological improvement followed by hospital discharge.

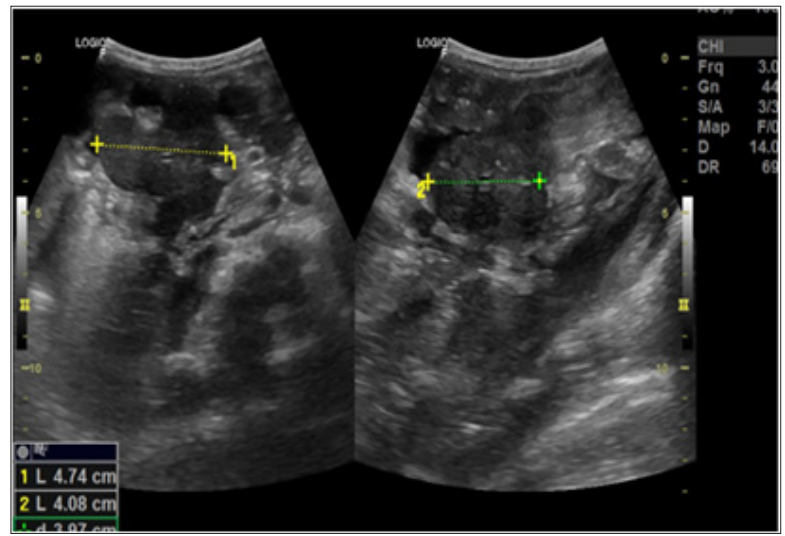

Figure 6: USG performed 15 days after starting treatment showing a volumetric reduction of lymph node mass in the right flank.

\section{Discussion}

Paracoccidioidomycosis is a systemic mycosis originates in Latin America, and its highest incidence is recorded in South America, notably in Brazil, Argentina, Colombia and Venezuela. In Brazil, the disease is endemic in the states of São Paulo, Rio de Janeiro, Minas Gerais, Rio Grande do Sul and Mato Grosso [1] mainly among rural populations, affecting males aged between 30 and 60 years-old and related to agricultural activities. The etiologic agent is a thermodymorphic fungus called Paracoccidioides brasiliensis. The entrance of the fungus is the upper respiratory tract, through inhalation of fungus spores, being the upper airways and lungs, the first sites affected, but due to hematogenous or lymphatic dissemination, any organ or system of the body can be affected. The main organs affected are the lungs, lymphatic system, adrenals, bones, digestive tract and central nervous system. According to the Brazilian Consensus on Paracoccidioidomycosis, the disease is classified, according to clinical data and its natural history, into: paracoccidioidomycosis infection; paracoccidioidomycosis disease; acute / subacute childhood / adolescence; chronic form of the adult: unifocal and multifocal; and residual disease [3].

Very rarely, paracoccidioidomycosis occurs in children under 7 years old [4]. According to the literature, the acute / subacute clinical form of paracoccidioidomycosis represents $3-5 \%$ of the cases [2]. The main manifestations of this clinical form are superficial and deep lymph node enlargement, suppurative lymph node masses, hepatosplenomegaly and various symptoms (digestive, cutaneous and osteoarticular), as well as anemia, fever and edema, with rapid deterioration of the general state of the child. Pulmonary and mucosal involvement are rare $[3,4]$. The present case occurred in a 6-year-old child living in an endemic zone, who began the clinical condition with non-specific gastrointestinal symptoms and palpable abdominal mass. It presented intra-abdominal lymph node masses in the imaging exams; anemia (Hematocrit $30.6 \%$ and Hemoglobin $10 \mathrm{~g} \%$ ), hepatomegaly, fever, asthenia and an important decrease of the general condition in $48 \mathrm{~h}$. Nonspecific clinical manifestations such as fever, malaise, weight loss and asthenia are reported by 40 to $80 \%$ of children with mycosis [5].

Intestinal functional changes and compression by lymph node masses can cause abdominal pain in colic, sometimes simulating acute surgical abdomen, in addition to prolonged diarrhea, nausea, vomiting or intestinal constipation $[4,6]$. The child in the case had constipation and palpable abdominal mass, after a CT scan, the surgical abdomen was ruled out. In childhood, the estimated time between infection and onset of symptoms is a few months [7]. In children and adolescents, paracoccidioidomycosis often manifests as a moderate or severe systemic disease, with a relatively short course and death occurs in approximately $10 \%$ of cases [4]. The report of having been bitten by a tick 72 hours before the onset of symptoms made it thought like the initial diagnosis the various conditions transmitted by this arachnid, but the child did not show rashes, which is frequent in most tick-borne infections.

The main differential diagnosis of paracoccidioidomycosis is pulmonary tuberculosis, in some cases occurring simultaneously in the same individual, as well as, ganglionar tuberculosis, atypical mycobacterioses, sarcoidosis, collagenosis and other systemic mycoses such as histoplasmosis and coccidioidomycosis $[4,8,9]$. In the case reported, differential diagnosis was made with lymphoproliferative disorders and ganglionar tuberculosis due to lymph node involvement. The imaging methods used, computed tomography and ultrasonography, allowed a better evaluation of the alterations found, but these disorders were nonspecific, able to 
simulate both neoplastic lesions and chronic infections. The gold standard for the definitive diagnosis is the finding of the fungus in a tissue sample [9]. The Potassium Hydroxide $(10 \% \mathrm{KOH})$ fresh smear test for direct fungus screening is also effective and cheaper for sputum examination, skin and mucosal scrapings, and ganglion aspirates [1].

The Grocott-Gomori Methenamine Silver (GMS) staining technique and the Periodic acid-Schiff (PAS) reaction are employed to evidence the fungus. Sabouraud agar culture should always be requested as a diagnostic method, although the growth of Paracoccidioides brasiliensis is slow [1,2]. The treatment of systemic disease can be done with amphotericin B. Trimethoprimsulfamethoxazole, itraconazole and other azole drugs may be used as alternatives. It is important to emphasize that the treatment of children and adolescents should be initiated ideally in the first months of the symptoms onset, when there is less visceral impairment and better prognosis $[2,4,9,10]$. The maintenance therapy is performed for a prolonged period of time, until cure criteria are obtained, aiming to reduce the risk of disease recurrence [9].

\section{Conclusion}

Paracoccidioidomycosis is a systemic mycosis endemic in some regions of Brazil. It is rare in the age group below 14 years-old, representing about $3-5 \%$ of cases. The main clinical manifestations in this age group are superficial and deep lymph node enlargement, suppurative lymph node masses, hepatosplenomegaly and various digestive, cutaneous and osteoarticular symptoms, as well as anemia, fever and emaciation, with rapid deterioration of the general state of the child. Pulmonary and mucosal involvement is rare. In this case report, paracoccidioidomycosis was present in a 6-year-old child with non-specific gastrointestinal symptoms associated with palpable abdominal mass. The diagnosis was obtained by pelvic lymph node biopsy that demonstrated the infection by Paracoccidioides. The treatment was made with amphotericin B for 28 days during the hospital internment. After clinical and radiological improvement, she was discharged from hospital with Itraconazole for another 28 days.

\section{References}

1. Trad HS, Trad CS, Elias Junior J, Muglia VF (2006) Revisão radiológica de 173 casos consecutivos de paracoccidioidomicose. Radiol Bras 39(3): 175-179.

2. Wanke B, Aidê MA (2009) Chapter 6-Paracoccidioidomicose. J Bras Pneumol 35(12): 1245-1249.

3. Shikanai Yasuda MA, Telles Filho FQ Mendes RP, Colombo AR, Moretti MA (2006) Consenso de paracoccidioidomicose. Rev Soc Bras Med Trop 39(3): 297-310.

4. Nogueira MG, Andrade GM (2015) Paracoccidioidomicose em crianças e adolescentes. Revista Medica De Minas Gerais 25(2): 260-268.

5. Nogueira MG, Andrade GM, Tonelli E (2006) Clinical evolution of paracoccidioidomycosis in 38 children and teenagers. Mycopathologia 161(2): 73-81.

6. Londero AT, Gonçalves AJ, Cruz ML, Rozembaum R, Cunha RQ et al. (1987) Paracoccidioidomicose disseminada infanto juvenil em adolescentes. Arq Bras Med 61(1): 5-12.

7. Londero AT, Melo IS (1983) Paracoccidioidomycosis in childhood. A Critical Review. Mycopathologia 82: 49-55.

8. Costa MA, Carvalho TN, Araújo Júnior CR, Borba AO, Veloso GA, et al. (2005) Manifestações extrapulmonares da paracoccidioidomicose. Radiol Bras 38(1): 45-52.

9. Palmeiro M, Cherubini K ,Yurgel L (2005) Paracoccidioidomicose Revisão da Literatura. Scientia Medica, Porto Alegre: PUCRS 15(4).

10. Paniago AM, Aguiar JI, Aguiar ES, Cunha RV, Gracy Regina de Oliveira LP et al. (2003) Paracoccidioidomicose: estudo clínico e epidemiológico de 422 casos observados no Estado de Mato Grosso do Sul. Rev Soc Bras Med Trop 36(4): 455-459.
ISSN: 2574-1241

DOI: 10.26717/BJSTR.2019.12.002311

Mônica Silva Costa Janson Ney. Biomed J Sci \& Tech Res

(C) (P) This work is licensed under Creative

Commons Attribution 4.0 License

Submission Link: https://biomedres.us/submit-manuscript.php

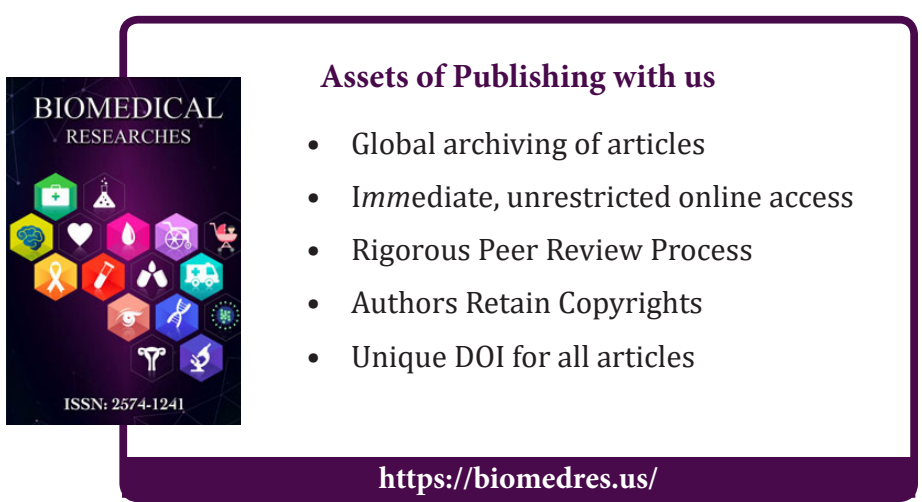

